\title{
Las enfermedades cardio-cerebro-vasculares en Medellín - Colombia, entre 2000- 2013: ¿un referente para las políticas públicas en salud?
}

\author{
Cardio-cerebrovascular diseases in Medellin - Colombia between 2000 and 2013: ¿̇a reference for public policy in \\ health?
}

María Victoria López-L1, María del Pilar Pastor-D22, Gustavo Alberto Arango-T33, Yesica Restrepo-T4

1 Socióloga, Magister en Medicina Social Universidad Autónoma Metropolitana - México. Docente Facultad de Enfermería Universidad de Antioquia, Investigadora Grupo Políticas Sociales y Servicios de Salud. Medellín Colombia, e-mail: mariavlopezlogmail.com

2 Enfermera, Doctora en Ciencias de la Salud Pública. Docente Facultad de Enfermería Universidad de Antioquia, Investigadora Grupo Políticas Sociales y Servicios de Salud. Medellín, Colombia. e-mail: ppastordurango@gmail.com

3 Sociólogo, Magister en Ciencias Sociales Facultad Latinoamericana de Ciencias Sociales -FLACSO México. Docente Facultad de Enfermería Universidad de Antioquia. Medellín, Colombia. e-mail: arangogus@gmail.com

4 Estudiante enfermería Universidad de Antioquia. Joven investigadora, integrante Grupo Políticas Sociales y Servicios de Salud. Medellín, Colombia. e-mail: yesica9317@hotmail.com

López-L MV, Pastor-D MP, Arango-T GA, Restrepo-T Y. Las enfermedades cardio-cerebro-vasculares en Medellín - Colombia, entre 2000-2013: ¿un referente para las políticas públicas en salud?. Univ. Salud. 2016;18(2):232-245. DOI: http://dx.doi.org/10.22267/rus.161802.34

\section{Resumen}

Introducción: Las políticas públicas son asuntos clave de la gestión gubernamental y su estudio es importante cuando se analiza el impacto que tienen frente a problemas relevantes que se reflejan en el perfil epidemiológico. Objetivo: Analizar la importancia que las enfermedades cardio-cerebro-vasculares tuvieron en la formulación de las políticas públicas en salud en Medellín entre 2000-2013. Materiales y métodos: Enfoque cualitativo con estrategia metodológica de análisis documental de corte hermenéutico. Se tomaron como base los acuerdos aprobados por el Concejo de Medellín y se seleccionaron aquellos, que tienen relación directa o indirecta con estas enfermedades, asimismo se revisaron planes de desarrollo contextualizados en lineamientos de políticas nacionales e internacionales. Resultados: Se encuentra un acuerdo que se refiere explícitamente a estas enfermedades. La mayoría son políticas orientadas, a grupos específicos o problemáticas relacionadas con promoción de la salud y prevención de las enfermedades cardio-cerebro-vasculares. El seguimiento y evaluación son fases menos tenidas en cuenta en el ciclo de las políticas públicas. Conclusiones: Los resultados sugieren la necesidad de una concepción amplia de la salud que oriente la formulación de las políticas locales e impacte un problema tan complejo e importante para el municipio, como lo son estas enfermedades.

Palabras clave: Políticas públicas; promoción de la salud; formulación de políticas; factores de riesgo. (Fuente: DeCS, Bireme).

\begin{abstract}
Introduction: Public policy is a key element to government administration and its study is important when analyzing the impact it has on relevant issues evidenced in the epidemiological profile. Objective: To analyze the importance of cardio-cerebrovascular diseases in the development of public policy in health in Medellin between 2000 and 2013.
\end{abstract}


Materials and methods: A qualitative approach with hermeneutic documentary analysis was done. The agreements approved by the Medellin council were taken as a base, from which those directly or indirectly related with these diseases were selected. Plans of action based on national and international policy guidelines were also reviewed. Results: An agreement that explicitly referred to these diseases was found. Most policy is oriented toward specific groups or issues related to health promotion and the prevention of cardio-cerebrovascular diseases. Follow-up and evaluation are the most overlooked stages of the public policy cycle. Conclusions: These results suggest the need for a broader definition of health that foments local policy development and impacts such a complex and important issue for the city by taking into account these diseases.

Keywords: Public policies; health promotion; policy making; risk factors. (Source: DeCS, Bireme).

\section{Introducción}

Este trabajo forma parte de los resultados de la investigación "Lógicas e interrelaciones en las políticas públicas orientadas al control de las enfermedades cardio-cerebro vasculares en Medellín 2000-2012", (Código Institucional de aprobación CODI-Enfer-01-2014), cuyo objetivo fue el de comprender las lógicas y articulaciones que subyacen en las políticas públicas relacionadas con las enfermedades cardiocerebro-vasculares en el municipio de Medellín, desde la revisión de los acuerdos municipales y los planes de desarrollo, así como desde la percepción de los actores sociales implicados en su formulación.

En Colombia a partir de la promulgación de la Constitución Política de 1991 y con la implementación del Sistema General de Seguridad Social en Salud en 1993, se ha venido reconociendo la salud como un servicio y un derecho de toda la población, que impacta en la organización y prestación de los servicios de salud y en la garantía de este derecho. En el contexto sociopolítico actual del país, las administraciones locales y los concejos municipales, son entes que proponen y aprueban las políticas públicas orientadoras del accionar municipal y el presupuesto necesario para ello. En tal sentido, ambas instituciones se constituyen en responsables directos del diseño, la planeación e implementación de políticas, programas y proyectos, siguiendo las directrices de los artículos 286, 287, 311 y 312 de la Constitución Política $^{1}$ y las normas complementarias que declaran que los municipios son entidades territoriales que gozan de autonomía para la gestión de sus intereses y para gobernarse por autoridades propias.

Las enfermedades cardio-cerebro-vasculares (ECCV) están incluidas en el grupo de las enfermedades no transmisibles y se constituyen en una problemática epidemiológica y de salud pública, al ser las principales causas de defunción en todo el mundo. Ellas afectan en mayor medida los países de ingresos bajos y medios $^{2}$ y sus consecuencias traen consigo, niveles significativos de discapacidad, deterioro de la calidad de vida y altos costos de atención en salud.

De acuerdo con el Plan de Acción 2008-2013 de la Organización Mundial de la Salud ${ }^{3}$, dada la importancia de estas enfermedades es necesario desplegar una estrategia mundial orientada a fortalecer intervenciones en prevención y control de estas enfermedades, dando prioridad al diseño de políticas relacionadas con la promoción de acciones para reducir los principales factores de riesgo modificables, controlar los factores determinantes de estas enfermedades y evaluar los avances en los ámbitos nacional, regional y mundial. Para hacer frente a esta realidad se sugiere valorar el riesgo y realizar acciones que contribuyan a su disminución, centrar la atención en la prevención primaria y secundaria que orientan las normas relacionadas con la investigación y la práctica clínica y desplegar las medidas necesarias para la detección y el manejo oportuno y eficaz de los factores que amenazan la salud cardio-cerebro-vascular.

En este contexto se insiste en reconocer que muchos de estos factores de riesgo están relacionados con estilos de vida, en particular el consumo excesivo o insuficiente de alimentos 
grasos, azucarados o salados, el sedentarismo, tabaquismo y el consumo excesivo de alcohol. El sobrepeso y la obesidad generan cambios metabólicos adversos como el aumento de la tensión arterial, los niveles de colesterol y una mayor resistencia a la insulina; además, provocan cardiopatías y accidentes cerebrovasculares, entre otros padecimientos 4 .

En Colombia las ECCV constituyen la mayor carga de morbilidad y mortalidad y de acuerdo con algunos autores ${ }^{5-7}$, están altamente relacionadas con el envejecimiento, estilos y hábitos de vida no saludables, como son las dietas malsanas, la inactividad física, el consumo o exposición al humo de tabaco y el uso nocivo del alcohol. En los índices de mortalidad estas causas aportan el $28.7 \%$ de las defunciones por todas las causas. En los hombres predominan la enfermedad coronaria y la enfermedad cardiaca isquémica y en las mujeres, las enfermedades relacionadas con la hipertensión arterial (enfermedad cerebrovascular e insuficiencia cardiaca) ${ }^{8}$.

El comportamiento de estas enfermedades en el municipio de Medellín es similar al observado en el país, desde el 2005 hasta el 2012 están situadas en las primeras diez causas de muerte: enfermedades isquémicas del corazón, enfermedades cerebrovasculares e hipertensivas y de acuerdo con el Plan de Salud Municipal 20122015 , la mortalidad por enfermedades isquémicas del corazón ha tenido una tendencia ascendente en la última década $(62,8$ casos por 100000 habitantes en el año 2.000, hasta 72,01 en el 2010) y en respuesta a esta problemática, se han formulado algunas políticas orientadas a su disminución y control ${ }^{9}$.

Respecto a las políticas públicas vale la pena destacar que éstas se conforman mediante un conjunto de acciones y decisiones orientadas a resolver de manera puntual, desde la gestión pública, un problema considerado público. En el análisis de estas políticas, es necesario reconocer el curso de acción ${ }^{10}$ que sigue un actor o un conjunto de actores para posicionar un problema como asunto de asunto de interés público susceptible de transformar. Es por ello, que se asume que las políticas públicas dan cuenta del tejido social entendido como proceso de mediación entre los gobernantes y diferentes actores sociales ${ }^{11}$. En tal sentido, ellas se expresan a través de decisiones, acciones y omisiones ${ }^{12}$, generadas por distintos actores clave y pasan por un proceso que va desde el diseño hasta su implementación y evaluación, lo que se define como ciclo de la política. Esta noción de las políticas como proceso relacional, orienta a entender como lo sugiere Aguilar ${ }^{13}$, que más allá de los discursos, las políticas públicas existen en la medida que las instituciones estatales asumen total o parcialmente el propósito de alcanzar los objetivos deseables o necesarios, a través de un proceso orientado a cambiar un estado de cosas reconocido problemático.

Estas políticas a la vez que instrumento para enfrentar situaciones problemáticas priorizadas, son elementos de legitimación de los gobernantes. Según Pal14, la lógica de las políticas públicas puede trascender la voluntad de quien la diseña y ejecuta, llegando, en ocasiones, a suceder que los gobiernos formulen de manera poco explícita los objetivos o los desconozcan, produciendo como consecuencia efectos opuestos a lo esperado.

La política no siempre se ejecuta como se formula debido a la influencia de las características sociales, económicas y culturales que orientan diferentes niveles de concreción y resultados disímiles, particularmente visibles en las localidades como espacio de las necesidades concretas. El concepto de lo público en referencia a la comprensión de las políticas constituye una preocupación nodal. A este respecto Arendt ${ }^{15}$,propone pensar que la esfera de lo público coincide con la de lo político al ser el espacio en el que predomina el bien común, la concreción de la polis o lugar en el que las personas concurren como iguales en torno a lo económico, lo socio-político, lo ambiental y lo cultural.

Coherente con lo anterior, en este artículo las políticas públicas se entienden como lineamientos clave de la gestión gubernamental, porque constituyen propuestas de solución a problemáticas reconocidas socialmente para las 
cuales se asignan recursos, se establecen mecanismos para su diseño, ejecución, seguimiento y evaluación definidos por la interlocución de actores gubernamentales y ciudadanos, lo que da origen a un patrón de comportamiento entre dichos actores ${ }^{16}$. En el campo de la salud, las políticas son una expresión de la importancia que los actores políticos dan al proceso salud-enfermedad-atención-muerte, así como a los distintos procesos que inciden en las condiciones de vida entendidas como conjunción de oportunidades, potencialidades y realizaciones.

Es por ello que tomando en cuenta la importancia que tienen las enfermedades cardio-cerebrovasculares (ECCV) en el perfil epidemiológico de Medellín, en este artículo se analiza la importancia de dicha problemática en la formulación de las políticas públicas en salud en el municipio durante el periodo 2000-2013. Lo anterior, es un aporte en la concreción del ejercicio de veeduría y control que requieren estas políticas, puesto que tienen implicaciones en las respuestas estatales, sociales e individuales, no solo para la atención a la salud, sino también para la promoción y prevención de la aparición de ECCV, así como para su tratamiento.

\section{Materiales y métodos}

Este artículo se enmarca en un enfoque cualitativo de investigación, con una estrategia metodológica de revisión y análisis documental de corte hermenéutico ${ }^{17,18}$. Las fichas de contenido y el análisis cruzado de las políticas públicas orientadas a la atención de las enfermedades objeto de estudio, facilitaron la interpretación a partir del consenso entre investigadores de diferentes disciplinas relacionadas con las ciencias sociales y la salud. Los lineamientos éticos indicados para la investigación social fueron un referente permanente durante el proceso investigativo; en el manejo de los documentos se tuvieron todos cuidados requeridos para evitar su alteración, mantenerlos a disposición de la población que los necesite y respetar los derechos de autor.
También, se contó con el aval del Comité de Ética en Investigación de la Facultad de Enfermería Universidad de Antioquia.

Para el análisis, se tomaron como base los acuerdos aprobados por el Concejo de Medellín y con el fin de ampliar la información precedente de ellos, se revisaron los proyectos de acuerdo y las actas de discusión de estas propuestas. Asimismo, se tuvieron en cuenta los planes de desarrollo municipal19-23, contextualizados en los lineamientos de Políticas Públicas NacionalesPlanes de Desarrollo Nacional y documentos del Consejo Nacional de Política Económica y Social CONPES ${ }^{24-26 .}$

En el análisis documental se siguieron orientaciones propuestas por Galeano ${ }^{27}$, que contempla, entre otras, las acciones de rastreo, inventario, muestreo de documentos existentes y disponibles, clasificación, selección, contextualización, valoración, análisis e interpretación, lectura cruzada y comparativa de los documentos, establecimiento de categorías de análisis, elaboración de memos analíticos y triangulación con otras fuentes y técnicas entre las cuales vale resaltar la recuperación de las voces de actores políticos comprometidos en el diseño, como ponentes y proponentes de los acuerdos.

El rastreo, inventario y muestreo de documentos existentes, se realizó mediante la revisión de las políticas públicas que contienen información directa o indirectamente relacionada con las ECCV, disponibles en bases de datos virtuales (web de la Administración Municipal de Medellín y el Concejo de Medellín) y en la biblioteca oficial Carlos Mauro Hoyos del Concejo de Medellín. El análisis de los documentos se hizo a partir de una matriz que clasifica cada acuerdo según su objeto de estudio, población beneficiaria, vigencia, concejales proponentes y ponentes, actores gubernamentales o sociales participantes, temas, períodos, resultados esperados y estrategias de evaluación, con el fin de hacer un filtro para la selección de los acuerdos pertinentes.

Con base en lo anterior, se seleccionaron 57 acuerdos en función de la relación explícita o 
implícita con las ECCV y aquellos que se corresponden con la normatividad propia del Concejo. En relación explicita, se aprueba el Acuerdo No. 3 de $2007^{28}$ que es la política pública para la prevención de la morbilidad y mortalidad por causas cardio-cerebro-vasculares. Los que tienen relación implícita son los que, sin un propósito específico frente a este tipo de enfermedades, inciden en su prevención a través de estilos de vida saludable, intervienen en el control de los factores de riesgo, o buscan mejorar las condiciones de vida para personas con posibles secuelas de las ECCV.

Respecto a la normatividad propia del Concejo, se analizaron los que brindan orientaciones para el diseño, la evaluación o el seguimiento de las políticas.

\section{Resultados}

Los principales responsables de la formulación de las políticas relacionadas con las ECCV son el Concejo y la Administración Municipal. En la revisión de los acuerdos considerados pertinentes sólo se encontró registro de una propuesta generada por la población. (Acuerdo 44 de 2010) ${ }^{29}$.

Orientados al análisis de la importancia de las ECCV en el ciclo de las políticas públicas, los resultados se presentan en función de tres ejes: el contexto de las políticas de salud, el Acuerdo 03 de 2007 por el cual se crea la política pública para la prevención de la morbimortalidad por causas cardio-cerebro-vasculares en el municipio de Medellín y la relación entre las políticas municipales en salud y el control de las ECCV.

\section{Contexto de las políticas de salud}

En este apartado se describen algunos referentes normativos nacionales e internacionales en los que se enmarca la formulación de las políticas locales en salud; así como las características de los acuerdos en tanto grupos poblacionales específicos, problemáticas priorizadas, intervenciones colectivas relacionadas con la prevención de las ECCV y la promoción de la salud. (Tabla 1).

Tabla 1. Clasificación de acuerdos relacionados con las ECCV según eje de la política. Medellín, 2000-2013

\begin{tabular}{|c|c|c|c|}
\hline Eje de política & $\begin{array}{l}\text { Población o } \\
\text { campo de } \\
\text { intervención }\end{array}$ & Objeto del acuerdo & $\mathbf{N}^{\circ}$ Acuerdo \\
\hline \multirow[t]{8}{*}{ Grupo poblacional } & $\begin{array}{l}\text { Niños, niñas, } \\
\text { adolescentes y } \\
\text { jóvenes }\end{array}$ & $\begin{array}{l}\text { Se institucionaliza plan de atención integral a la infancia y se crea } \\
\text { el concejo de política de infancia en la ciudad de Medellín }{ }^{30} \text {. }\end{array}$ & $013 / 2001$ \\
\hline & & $\begin{array}{l}\text { Se crea el Programa "Buen Comienzo" para promover el desarrollo } \\
\text { integral, diverso e incluyente de los menores de } 6 \text { años }^{31} \text {. }\end{array}$ & $014 / 2004$ \\
\hline & & $\begin{array}{l}\text { Se adopta la Política Pública de Atención Integral a la Primera } \\
\text { Infancia Buen Comienzo, se desarrolla un Sistema de Atención } \\
\text { Integral y se modifica el Acuerdo } 14 \text { de } 2004^{32} \text {. }\end{array}$ & $058 / 2011$ \\
\hline & & $\begin{array}{l}\text { Se crea el programa UNIRES Unidades Integrales de Renovación } \\
\text { social en el Municipio de Medellín }{ }^{33} \text {. }\end{array}$ & $007 / 2003$ \\
\hline & & Se crea el programa Comités de Salud Estudiantil en Medellín ${ }^{34}$. & $008 / 2005$ \\
\hline & & $\begin{array}{l}\text { Se adopta el plan Estratégico Municipal de Desarrollo Juvenil de } \\
\text { Medellín 2007- } 2015^{35} \text {. }\end{array}$ & $076 / 2006$ \\
\hline & Mujeres & $\begin{array}{l}\text { Se expide la Política Pública para las mujeres Urbanas y Rurales del } \\
\text { Municipio de Medellín }{ }^{36} \text {. }\end{array}$ & $022 / 2003$ \\
\hline & & 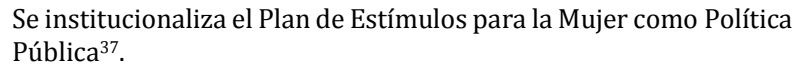 & $033 / 2005$ \\
\hline
\end{tabular}




\begin{tabular}{|c|c|c|c|}
\hline & Adultos mayores & $\begin{array}{l}\text { Se adopta la política pública para la población de la tercera edad } \\
\text { en Medellín }{ }^{38} \text {. }\end{array}$ & $018 / 2001$ \\
\hline & & Se institucionaliza el Foro Anual del Adulto Mayor en Medellín ${ }^{39}$. & $084 / 2006$ \\
\hline & & $\begin{array}{l}\text { Se institucionaliza el programa "Canas al aire" Concejo de } \\
\text { Medellín }{ }^{29} \text {. }\end{array}$ & $044 / 2010$ \\
\hline \multirow[t]{8}{*}{ Problemática } & Discapacidad & $\begin{array}{l}\text { Acondiciónese las estaciones del Metro para que sean accesibles a } \\
\text { personas con discapacidad }{ }^{40} \text {. }\end{array}$ & $002 / 2001$ \\
\hline & & Se institucionaliza la "Semana de la Discapacidad" 41. & $033 / 2004$ \\
\hline & & $\begin{array}{l}\text { Se crea el Programa "Apoyo técnico para la Movilidad" } \\
\text { en procura de la integración de las personas con discapacidad }{ }^{42} \text {. }\end{array}$ & $053 / 2007$ \\
\hline & & $\begin{array}{l}\text { Se adopta la política pública en discapacidad para el municipio de } \\
\text { Medellín }{ }^{43} \text {. }\end{array}$ & $086 / 2009$ \\
\hline & Consumo tabaco & $\begin{array}{l}\text { Se prohíbe el consumo de cigarrillo, tabaco y sus derivados en los } \\
\text { establecimientos oficiales municipales de atención al público, en } \\
\text { oficinas estatales del municipio y en edificios de propiedad del } \\
\text { Municipio de Medellín }{ }^{44} \text {. }\end{array}$ & $050 / 2006$ \\
\hline & Desnutrición & $\begin{array}{l}\text { Se establece la Política Pública de Seguridad y Soberanía } \\
\text { Alimentaria y Nutricional en Medellín }{ }^{45} \text {. }\end{array}$ & $038 / 2005$ \\
\hline & & $\begin{array}{l}\text { Se crea el programa de recuperación nutricional en la ciudad de } \\
\text { Medellín }{ }^{46} \text {. }\end{array}$ & $056 / 2008$ \\
\hline & $\begin{array}{l}\text { Enfermedad cardio- } \\
\text { cerebro-vascular }\end{array}$ & $\begin{array}{l}\text { Se adopta la Política pública para la prevención de la } \\
\text { morbimortalidad por causas cardio-cerebro-vasculares en el } \\
\text { municipio de Medellín }{ }^{28} \text {. }\end{array}$ & $003 / 2007$ \\
\hline \multirow[t]{6}{*}{$\begin{array}{l}\text { Intervenciones } \\
\text { colectivas }\end{array}$} & Ciudad saludable & $\begin{array}{l}\text { Se implementa en el Concejo de Medellín, el programa } \\
\text { Participación interdisciplinaria de investigación en temas de } \\
\text { ciudad }^{47} \text {. }\end{array}$ & $013 / 2003$ \\
\hline & & $\begin{array}{l}\text { Se implementa en forma permanente el Programa Medellín } \\
\text { Saludable }{ }^{48} \text {. }\end{array}$ & $018 / 2003$ \\
\hline & & $\begin{array}{l}\text { Se crea la Red de atención de urgencias para el municipio de } \\
\text { Medellín }{ }^{49} \text {. }\end{array}$ & $021 / 2004$ \\
\hline & & $\begin{array}{l}\text { Se crea en el Municipio de Medellín las " Unidades Móviles de } \\
\text { Salud"50. }\end{array}$ & $059 / 2008$ \\
\hline & $\begin{array}{l}\text { Recreación y } \\
\text { deporte }\end{array}$ & $\begin{array}{l}\text { Se institucionaliza la Semana del Deporte La Recreación y La } \\
\text { Lúdica }^{51} \text {. }\end{array}$ & $072 / 2006$ \\
\hline & Movilidad & $\begin{array}{l}\text { Se reglamenta el Sistema de Bicicletas en la ciudad de Medellín que } \\
\text { hará parte del Plan de movilidad de la ciudad }{ }^{2} \text {. }\end{array}$ & $084 / 2009$ \\
\hline
\end{tabular}

Nota: Se excluyen los acuerdos que se refieren a planes de desarrollo, ejecutorias presupuestales y normatividad del Concejo

Desde el punto de vista normativo todos los acuerdos seleccionados están enmarcados en directrices nacionales emanadas de la Constitución Política de 1991 y de leyes específicas, ya se trate de normas que posibilitan las ejecuciones o adiciones presupuestales, orientaciones tendientes a la organización y el funcionamiento de los municipios o de garantía de derechos para la protección y la asistencia de las personas.
En los acuerdos analizados aparecen de manera explícita las directrices internacionales y nacionales que se tuvieron en cuenta para su diseño, tales como la Convención Mundial sobre los Derechos del Niño en 1989 que permitió en Colombia la aprobación de la Ley 12 de 1991, el "Plan de Acción a favor de la Infancia 1991-1994" (PAFI) y la Ley 1098 de 2006 "Código de infancia y adolescencia”, concreción de los compromisos del país en la Cumbre Mundial sobre la Infancia realizada en 1990; la Red de Observatorios de 
América Latina; los compromisos internacionales surgidos en las Cumbres de Roma en 1996 y de La Habana en el 2003 en los que se acuerda disminuir el hambre al 2015; Convenio Marco de la OMS para el Control del Tabaco firmado en Ginebra en mayo de 2003, redes públicas de diferentes ciudades del mundo como Barcelona, Dublín, Copenhague, Washington D.C. para promover el uso de la bicicleta; la Clasificación Internacional del Funcionamiento de la Discapacidad y de la Salud, de la OMS y OPS aprobada en 2001; la Convención sobre los Derechos de las Personas con Discapacidad adoptados por la Asamblea General de las Naciones Unidas el 13 de diciembre de 2006 y ratificados por Colombia mediante la Ley 1346 del 31 de julio de 2009.

Respecto a las características de las políticas identificadas, algunos actores posicionan en sus motivaciones para el diseño y la concertación con el Concejo, la preocupación por grupos poblacionales específicos, como se describe a continuación:

Infancia, adolescencia y juventud. En actas de debate y en los acuerdos se planteó la necesidad de una política de infancia, con acciones de promoción de la salud y la prevención de las enfermedades, de tal manera que se eviten factores de riesgo y se promueva la calidad de vida de los niños en el marco de los derechos, el bienestar y la integración al entorno. En esta línea, se identificaron varios acuerdos relacionados entre sí por su intención de procurar un cuidado integral. En 2001 se institucionalizó el Plan de Atención Integral a la Infancia y creó el Consejo de Política de Infancia en la ciudad tomando en cuenta aspectos físicos, psíquicos y sociales y subrayando la importancia de acompañar a los menores con acciones de política para generar estilos de vida saludable a través de los diferentes ámbitos (salud, educación, saneamiento, sostenimiento básico y derecho de protección).

En el año 2004 se creó el Programa "Buen Comienzo" el cual, siguiendo los lineamientos del Acuerdo 13 de $2001^{30}$, mantiene la intención de promover un desarrollo diverso e incluyente y focaliza su accionar en los menores de 6 años. En
2011 con la "Política Pública de Atención Integral a la Primera Infancia Buen Comienzo", se recoge la orientación de los acuerdos anteriores en cuanto al compromiso con la salud, el bienestar de la primera infancia y la atención integral de los niños y las niñas, desde la gestación hasta los cinco años.

Así mismo, en el año 2003 se estableció el programa UNIRES Unidades Integrales de Renovación social en el Municipio de Medellín para brindar atención personalizada en educación especial, nutricional, médica y asistencial a población de 5 a 15 años, escolarizada en establecimientos educativos públicos de la ciudad. En el 2005 se aprobó el Programa Comités de Salud Estudiantil, para la promoción del cuidado y autocuidado de la salud por medio de estrategias de información, educación y comunicación.

El "Plan Estratégico Municipal de Desarrollo Juvenil de Medellín 2007- 2015”, se adoptó en 2006, con las siguientes líneas de acción: convivencia pacífica y democrática, inclusión social juvenil, empoderamiento juvenil, reconocimiento de prácticas y representaciones juveniles, inserción socio-laboral y productiva, promoción del talento juvenil para la empleabilidad y la gestión de iniciativas productivas y fortalecimiento de la construcción del conocimiento en, con y para la juventud en la ciudad.

Mujeres. A partir del reconocimiento de sus necesidades y derechos se propone garantizar su bienestar y el mejoramiento de su calidad de vida, con la intervención integral de los factores del entorno. En el año 2003 se aprobó la "Política Pública para las Mujeres Urbanas y Rurales del municipio de Medellín", la cual se complementó en el 2005 con el acuerdo que institucionalizó el "Plan de estímulos para la mujer" como política pública con su participación en programas formativos, laborales, de salud, de justicia, culturales, deportivos y recreacionales.

Adultos mayores. En 2001 se adoptó la política pública para la población de la tercera edad, la cual se implementa y desarrolla como 
responsabilidad del Comité Interinstitucional Pro-adulto mayor y en 2006, dicha política se fortalece con el Acuerdo No. 084 que institucionalizó el Foro Anual del Adulto Mayor en Medellín para promover la participación, integración y empoderamiento de líderes comunitarios. Por iniciativa de este grupo poblacional, en 2010 se institucionalizó el programa "Canas al aire".

En cuanto a los problemas priorizados que caracterizan el contexto de las políticas se destacan la situación de discapacidad, el tabaquismo, la desnutrición y las enfermedades cardio-cerebro-vasculares.

Situación de discapacidad. Es considerada una necesidad de respuesta política por parte de los concejales en busca del mejoramiento de la movilidad, la equiparación de oportunidades y el reconocimiento de derechos a personas que tienen alguna limitación permanente o temporal. En el año 2001 se formuló un acuerdo que propugna por la adecuación de las estaciones del metro en la ciudad buscando mejoramiento en la calidad de vida y bienestar para las personas discapacitadas.

En 2004 se institucionalizó la "semana de la discapacidad", una manera de sensibilizar a toda la población y posicionar esta problemática como realidad social, durante la cual se realizan eventos lúdico-educativos, culturales, sociales y deportivos. El programa "Apoyo Técnico para la Movilidad" se creó en el año 2007 para lograr la integración social de las personas con discapacidad. Estos acuerdos crearon condiciones para la formulación de la política pública en discapacidad en Medellín en el año 2009, en la cual se reconoce que la situación de discapacidad puede ser una secuela de las ECCV.

Tabaquismo. Con el fin de garantizar a los habitantes el derecho a vivir en un ambiente sano y promover espacios libres de humo, en 2006 se prohibió el consumo de cigarrillo, tabaco y sus derivados en los establecimientos oficiales municipales de atención al público y en oficinas estatales y edificios propiedad del Municipio de Medellín.
Desnutrición. La política pública de Seguridad y Soberanía Alimentaria y Nutricional se aprobó en 2005, la cual reconoce los derechos a la alimentación y a un entorno sano mediante el fortalecimiento del sector agrícola regional y el valor de la cultura alimentaria, con criterios de identidad y equidad ambiental y territorial. Como complemento a la política anterior, en 2008 se creó el programa de recuperación nutricional con el objetivo de evitar la muerte de los niños y niñas con desnutrición o con gran riesgo de desarrollarla.

Enfermedades cardio-cerebro-vasculares. De manera explícita, se reconocen como un problema de salud presente en el perfil epidemiológico, porque ocupa los primeros lugares entre las causas de enfermedad y de muerte. Por ello en el 2007 se aprobó el Acuerdo 03 que crea la política pública para la prevención de la morbilidad y la mortalidad por estas causas en Medellín. En el contexto también se destacan los acuerdos caracterizados por proponer intervenciones colectivas relacionadas con la prevención de las ECCV y la promoción de la salud, que se dirigen a la población en general e impactan su calidad de vida desde una perspectiva amplia, en componentes tales como salud, recreación, deporte y movilidad.

Salud. En el año 2003 se implementó en forma permanente el "Programa Medellín Saludable" que define salud integral como la resultante de la cultura, los estilos de vida, los buenos hábitos alimentarios y los ambientes sanos y saludables. Asimismo, para mejorar el acceso a los servicios de salud, se creó la Red de Atención de Urgencias para el municipio de Medellín en el año 2004 y las Unidades Móviles de Salud en el 2008. Por otro lado, en el 2003, con el programa Participación Interdisciplinaria en temas de ciudad, se promueve el desarrollo de propuestas investigativas que aporten de manera integral al bienestar de la población, involucrando problemáticas urbanas, dilemas sociales $y$ ambientales y evaluación de las diversas políticas públicas.

Recreación y deporte. En el año 2006 se institucionalizó la "Semana del Deporte, la 
Recreación y la Lúdica" como una estrategia de participación social que contribuye al mejoramiento de la calidad de vida, el fortalecimiento de estilos de vida saludable y la sensación subjetiva de bienestar, circunstancias que inciden en la prevención de la enfermedad y en la promoción de la salud.

Movilidad. En 2009 se reglamentó el Sistema de Bicicletas en la ciudad de Medellín, que propone un transporte alternativo como aporte a una movilidad urbana limpia, con reducción de los niveles de contaminación; además, promueve estilos de vida saludables, cultura ciudadana de civismo, respeto y convivencia.

Política pública para la prevención de la morbilidad y la mortalidad por ECCV. En el Informe de Ponencia de marzo 2007 para debate del Proyecto 307, aprobado en Acuerdo No.03 de 2007, se reconocen las ECCV como una problemática social de alta frecuencia según el perfil epidemiológico del municipio. Estas enfermedades $y$ sus factores asociados se identifican como una situación necesaria de intervenir, con atención especial a las mujeres, toda vez que una tercera parte de las muertes en este grupo, se debe a enfermedades asociadas a la menopausia, al síndrome de ovario poliquístico. También se menciona que durante los primeros días del ciclo menstrual hay mayor riesgo de ataque cardiaco.

En esta política se establece un compromiso con la prevención y el control de factores de riesgo, mediante el desarrollo de acciones individuales tales como el monitoreo de signos y síntomas asociados a dichas enfermedades. En cuanto a acciones colectivas, plantea la realización anual de la semana de promoción y prevención de las ECCV, el fortalecimiento de estilos de vida saludable a través de acciones educativas para favorecer la adecuada alimentación y el ejercicio físico y evitar el consumo de tabaco y alcohol; igualmente, incluye la práctica deportiva y el mejoramiento de la movilidad y de la red prestadora de servicios que facilite la atención en salud; para lograr lo anterior, se requiere la articulación intra e intersectorial.
En el Acuerdo No. 03 de 2007 no se explicitan acciones encaminadas al seguimiento y la evaluación, etapas del ciclo de la política que permiten identificar los avances en la implementación y hacer las actualizaciones pertinentes, de tal manera que se logren los objetivos y se contribuya a la transformación de la problemática inicial.

Políticas municipales en salud y control de las ECCV. La relación entre las características de los acuerdos permiten comprender que las ECCV hacen parte de la agenda pública, puesto que la aprobación de una política trasciende la formalización aislada del discurso sobre problemas prioritarios y grupos poblacionales y configuran interrelaciones sinérgicas para la solución de los problemas. Como se señaló, el Acuerdo No. 03 de 2007 se refiere explícitamente a las ECCV, sin embargo, la implementación de los demás acuerdos analizados, impacta en el comportamiento de dichas enfermedades por su perspectiva de promoción de la salud, de prevención o por su contribución en el largo plazo a la disminución de las complicaciones.

En este sentido, los acuerdos dirigidos al grupo de niños, niñas, adolescentes y jóvenes, impulsan el desarrollo de hábitos y comportamientos saludables, relacionados con la alimentación, la actividad física y el consumo de sustancias psicoactivas como estrategias de fomento a la salud y prevención de la enfermedad. En cuanto a las mujeres, se busca, además de lo anterior, facilitar la atención en salud y estimular el desarrollo de acciones en pro de la salud de sus hijos. Otros acuerdos buscan impactar el deterioro físico y mental de los adultos mayores, ocasionado no sólo por la edad sino también por las enfermedades prevalentes en este grupo, entre ellas las cardio-cerebro-vasculares.

Asimismo, se identifican acuerdos que promueven la salud en toda la población, fomentando el uso de la bicicleta, las prácticas deportivas y recreativas y el desarrollo de acciones que contribuyen a hacer de Medellín una ciudad saludable. Asimismo, se reconoce que la situación de discapacidad caracterizada por la movilidad reducida, es una de las consecuencias 
de las ECCV que amerita el desarrollo de acciones que favorezcan la inclusión de personas con estas secuelas para facilitar su desplazamiento en transporte público.

La descripción anterior supone un impacto de las políticas públicas en las condiciones de salud de la población del municipio cuando se avanza a su implementación. Por ello, algunos acuerdos proponen acciones tales como la gestión y evaluación de programas mediante la construcción de alianzas y redes en los distintos niveles; el desarrollo de estrategias de información, educación y comunicación; el estímulo a la participación ciudadana.

Así mismo, se propende por el mejoramiento del acceso a la atención pre-hospitalaria con la creación de la red de atención de urgencias y emergencias; el aumento de cobertura de servicios para poblaciones consideradas vulnerables con las unidades móviles de salud y la implementación de la estrategia de APSRenovada; todo ello bajo la responsabilidad de la administración municipal y la participación intersectorial de otros organismos estatales locales, regionales y nacionales, organizaciones no gubernamentales y empresas del sector privado.

Para hacer posible el desarrollo de estrategias de implementación, se aprobaron los planes de desarrollo municipal en los que se asignan recursos financieros para el desarrollo de acciones que favorezcan el control de las ECCV y garanticen el cumplimiento de competencias asignadas por ley a los municipios. Si bien es cierto que el acuerdo No. 03 de 2007 no precisa acciones para su seguimiento y evaluación, se incluye la vigilancia epidemiológica de la morbilidad y la mortalidad por estas causas como un componente básico. En otros acuerdos se establece la periodicidad del seguimiento y evaluación pero no definen criterios ni responsables.

\section{Discusión}

La formulación de políticas, programas y proyectos son respuestas a problemas socialmente reconocidos como las ECCV. Dada la complejidad de esta problemática en Medellín que afecta a individuos y colectivos, se proponen líneas de acción en distintos ámbitos para la promoción de la salud, la prevención de la enfermedad, la atención médica y la rehabilitación. En promoción de la salud se busca la intervención de múltiples factores relacionados con los estilos de vida, que son responsabilidad de diferentes sectores. Según la Organización Mundial de la Salud -OMS ${ }^{4}$ la carga de mortalidad, morbilidad y discapacidad por ECCV es creciente en los países en desarrollo y preocupante si se tiene en cuenta que allí, las personas son en promedio más jóvenes. Entre los factores de riesgo relacionados con estas enfermedades se destacan: la hipertensión arterial, la hipercolesterolemia, la escasa ingesta de frutas y hortalizas, el sobrepeso u obesidad, la falta de actividad física y el consumo de tabaco.

En el análisis de los acuerdos municipales analizados se reconocieron los puntos de partida de las políticas y sus cambios, como un proceso de construcción de consensos entre los actores. Para ello se siguieron lineamientos de autores que como Muller ${ }^{53}$ han acotado esta temática, a partir de la pregunta ¿cómo nacen y se transforman las políticas públicas? Las políticas revisadas, se formularon a partir de convenios y lineamientos nacionales e internacionales, entre los que se desatacan los Objetivos de Desarrollo del Milenio (ODM-2000) $)^{54}$, en cuanto a seguridad alimentaria y nutricional, la atención a la niñez y la equidad de género. También se reconoce la preocupación permanente de la OMS por el control de las Enfermedades Crónicas No Transmisibles (ECNT), entre ellas las ECCV, los factores de riesgo asociados y el fomento de hábitos saludables ${ }^{55}$.

Otro referente en el nacimiento de las políticas es el entorno local y la necesidad de priorizar grupos poblacionales que requieren intervenciones de largo plazo por los cambios demográficos que muestran un crecimiento de la población adulta mayor ${ }^{56}$ y una alta tasa de mortalidad en menores 
de 25 años atribuible al problema de la violencia desde fines de la década de 198057. Estas intervenciones se orientan principalmente a dos grupos de población: niños, niñas, adolescentes y jóvenes para formar hábitos saludables y adultos mayores para fomentar estilos de vida que prevengan sus complicaciones.

Del reconocimiento de estas características relacionadas con el origen de la política, surgen nuevas preguntas de investigación acerca de su alcance, si trascienden el periodo de gobierno, sirven como guía para la acción en el largo plazo, mejoran las condiciones de vida y contribuyen a la construcción de un tejido social más sólido, o sólo son la expresión de la voluntad de quienes la formulan ${ }^{58}$. En este sentido, es necesario tener en cuenta, como lo sugiere Stoker59, que la implementación de las políticas implica la interacción de los actores que impulsaron su formulación a partir de múltiples motivaciones, el reconocimiento de la realidad social y el despliegue de acciones efectivas que contribuyan a la transformación de la situación problemática.

Otro tópico de interés en el análisis de las políticas durante el período, se relaciona con su comprensión como proceso dinámico no lineal, que puede tener distintos puntos de inicio. La identificación de varias rutas en el curso de la política pública muestra que primero puede formularse como tal y luego se concreta en otros acuerdos cuyo objeto es el establecimiento de programas para su implementación o, en otros casos, se parte de la formulación de un plan, proyecto o intervención específica $y$ posteriormente, surgen acuerdos que formalizan la política pública.

Las políticas públicas analizadas responden a problemas estructurales, tienen vigencia en el tiempo $y$ se articulan con los planes gubernamentales y como lo proponen Arroyave ${ }^{60}$ y Roth ${ }^{61}$, incluyen la identificación del problema, la factibilidad de las acciones propuestas, el papel de la comunidad y la pertinencia de la política pública en la agenda administrativa. Esta es una línea de trabajo necesaria de ahondar en futuros trabajos en los que pueda ahondarse la vivencia de estas políticas desde la experiencia de otros actores.

Si bien este trabajo centró la mirada en la información proveniente de los actores gubernamentales, los resultados indican la importancia de contar con la participación, no solo a estos actores sino también con la población en general, dado que, las políticas en tanto sistema de acción pública, combina procesos de lo gubernamental y no gubernamental, en cuyos límites no son precisos el comienzo ni el final62. Este reconocimiento a la acción pública es una línea trabajo que debe profundizarse para lograr, con la presencia efectiva de la comunidad, la continuidad y la sinergia que requieren los procesos políticos ya que el problema cardiovascular necesita resonancia y articulación.

En esta misma dirección es preciso reconocer que esta problemática al igual que otros problemas de salud pública está determinada por condiciones económicas, sociales, culturales y ambientales y que la responsabilidad de los sistemas de salud, no se agota en las fronteras de acción de la autoridad y gestión local. En este caso los municipios, están llamados ampliar su campo de acción a través de la intersectorialidad y asumirse como escenario fundamental de gestión de la salud para la prevención y atención de problemas cardio-cerebro-vasculares, gestión esta que se implementa en consonancia con los programas nacionales de salud y necesita ajustarse a las posibilidades y necesidades del territorio ${ }^{63}$.

\section{Conclusiones}

Los acuerdos analizados sugieren la necesidad de una concepción amplia de la salud que oriente la formulación de las políticas locales e impacte un problema complejo e importante para el Municipio, como son las ECCV. Lo anterior es posible sí las motivaciones son compartidas entre quienes formulan y quienes ejecutan estas políticas; si estos últimos mantienen su esencia al ponerla en marcha porque consideran que ambas fases hacen parte de un mismo proceso; si se cumplen las condiciones de factibilidad incluidas 
en el diseño; si se hace seguimiento y evaluación para introducir los cambios pertinentes.

Es necesario enfatizar en el carácter público de las políticas como respuesta a necesidades sociales complejas, cuyas dinámicas no tienen actores, causas ni periodos de tiempo claramente delimitables; es por ello que para interpretar los aportes de las políticas municipales al control de las ECCV, se requiere comprender la complejidad de las lógicas que subyacen entre ellas y su relación con la realidad, asunto en el cual el carácter público de las acciones no se refiere de manera exclusiva al Estado, aunque sí es su responsabilidad central.

Por último, este trabajo permite reafirmar la importancia de leer las políticas desde el hacer de actores clave bajo una perspectiva histórica y la pertinencia que tiene, en el análisis de las políticas, acercarse a ellas con problemáticas concretas, pero a la vez con una posición teórica que permita el análisis de la relación de dicha políticas con otras; en el caso que nos ocupa la relación de las ECCV y otras referidas a la promoción de la salud y prevención de ellas.

Conflicto de intereses: Ninguno; el proyecto fue financiado por el Comité para el Desarrollo de la Investigación -CODI-UdeA.

\section{Referencias}

1. Colombia. Asamblea Nacional Constituyente. Constitución Política de Colombia 1991. [internet]. [consultado 2015 Jun 15]. Disponible en: http://www.alcaldiabogota.gov.co/sisjur/normas/Nor ma1.jsp?i=4125

2. World Health Organization. Global status report on noncommunicable disaeses 2010. [Internet]. [Consultado 2015 ene 29]. Disponible en: http://www.who.int/nmh/publications/ncd_report_ful l_en.pdf

3. Organización Mundial de la Salud, Organización Panamericana de la Salud. 28 ${ }^{\text {a }}$ Conferencia Sanitaria Panamericana 64a Sesión del Comité Regional. Estrategia para la prevención y el control de las enfermedades no transmisibles, 2012-2025. [Internet]. [Consultado 2015 ener 29]. Disponible en: http://www.msal.gov.ar/images/stories/bes/graficos/ 0000000202cnt-2013-07_estrategia-nacionalprevencion-control-ent-2012-2025.pdf
4. Organización Mundial de la Salud. Informe sobre la salud en el mundo 2002: reducir los riesgos y promover una vida sana. Ginebra, 2002.

5. Colombia. Instituto Nacional de Salud, Observatorio Nacional de Salud. Enfermedad cardiovascular principal causa de muerte en Colombia. [Internet]. [Consultado 2015 ene 28]. Disponible en: http://www.ins.gov.co/lineas-deaccion/ons/boletin\%201/boletin_web_ONS/boletin_01 _ONS.pdf)

6. Organización Mundial de la Salud. Informe sobre la situación mundial de las enfermedades no transmisibles Resumen de orientación. [Internet]. [Consultado 2015 ene 30]. Disponible en: http://www.who.int/nmh/publications/ncd_report_su mmary_es.pdf

7. Banco de la República. Documentos de trabajo sobre Economía Regional. [Internet]. [Consultado 2015 ene 29]. Disponible en: http://www.banrep.gov.co/docum/Lectura_finanzas/p df/dtser_209.pdf

8. Instituto Nacional de Salud. Las enfermedades cardiovasculares: un problema de salud pública y un reto global. Bogotá. Biomédica. 31(4):469-473.

9. Alcaldía de Medellín. Plan de Salud Municipal 20122015: Medellín, una ciudad saludable. Revista de la Secretaría de Salud Municipio de Medellín. 2012; 5(1):44.

10. Meny Y, Thoening JC. Las políticas públicas. Barcelona: Ariel; 1992.

11. Dye TR. Understanding Public Policy. Nueva Jersey, Estados Unidos: Prentice Hall; 2002

12. Anderson J. Public Policy Making: an Introduction. Boston. Mifflin Company. In: Howlett M, Ramesh M. Studying Public Policy. Policy Cicle and Policy Subsystems. Ontario: Oxford University Press; 1995.

13. Aguilar Villanueva LF. Marco para el análisis de las políticas públicas. En: Mariñez F, Garza V. (comps) Política pública y democracia en América Latina. Del análisis a la implementación. Primera edición. México D.F: Editorial Miguel Ángel Porrúa; 2009.

14. Pal L. Public Policy Analysis: An Introduction. Toronto: Methuen, 1987.

15. Arendt H. La Condición humana. $6^{\mathrm{a}}$ ed. Barcelona: Paidós; 1996:44-45.

16. Roth Deubel AN. Perspectivas teóricas para el análisis de las políticas públicas: ¿de la razón científica al arte retórico?. Estud. Polít. 2008(33):67-9.

17. Galeano ME. Investigación documental: una estrategia no reactiva de investigación social. En: Galeano ME. Estrategias de investigación social cualitativa: El giro en la mirada. Primera edición. Medellín: La Carreta Editores; 2004:113-144.

18. Gómez-Mendoza MÁ. Análisis de contendido cualitativo y cuantitativo: Definición, clasificación y metodología. Rev Cien Hum. 1999;6(20):103-113.

19. Plan de Desarrollo 1998-2000 "Medellín Competitiva". Medellín. 1998.

20. Medellín. Plan de Desarrollo Municipal 2001-2003: "Medellín, la ciudad de las oportunidades". Medellín. 2001. 
21. Municipio de Medellín. Plan de Desarrollo. Plan de desarrollo 2004-2007 "Medellín, compromiso de toda la ciudadanía". [Internet]. [Consultado 2015 oct 28]. Disponible https://www.medellin.gov.co/irj/go/km/docs/pccdesi gn/SubportaldelCiudadano_2/PlandeDesarrollo_0_0_0/ Shared\%20Content/pdf\%20codigo\%20buen\%20comi enzo/Texto\%20Completo\%20Acuerdo\%20Plan.pdf

22. Municipio de Medellín. Plan de Desarrollo 2008-2011 "Medellín es Solidaria y Competitiva". Gaceta Oficial Gaceta №3261. [Internet]. [Consultado 2015 oct 28]. Disponible

en: www.medellin.gov.co/irj/go/km/docs/wpccontent/Sit es/Subportal\%20del\%20Ciudadano/Plan\%20de\%20D esarrollo/Secciones/Publicaciones/Documentos/Gacet a\%200ficial\%20Plan\%20de\%20Desarrollo.pdf

23. Alcaldía de Medellín. Plan de Desarrollo Medellín 2012 - 2015: Medellín Un Hogar para la Vida. [Internet]. [Consultado 2015 oct 28]. Disponible en: https://www.medellin.gov.co/irj/go/km/docs/wpcco ntent/Sites/Subportal\%20del\%20Ciudadano/Plan\%20 de\%20Desarrollo/Secciones/Publicaciones/Document os/PlaDesarrollo2012-2015/2012-04-

30_Proyecto\%20de $\% 20$ acuerdo\%20VERSION\%20CO MPLETA.pdf

24. Colombia. Departamento Nacional de Planeación. Planes de Desarrollo anteriores. Texto de Planes de Desarrollo de Años Anteriores. [Internet]. [Consultado 2015 oct 7]. Disponible en: https://www.dnp.gov.co/Plan-Nacionalde-Desarrollo/Paginas/Planes-de-Desarrolloanteriores.aspx

25. Colombia. Departamento Nacional de Planeación. Documentos CONPES. [Internet]. [Consultado 2015 oct 7]. Disponible en: https://www.dnp.gov.co/CONPES/documentos-conpes

26. Colombia. Departamento Nacional de Planeación, Sistema de seguimiento a documentos Conpes (SISCONPES). Documentos Conpes aprobados. Disponible en: https://sisconpes.dnp.gov.co/DocumentosConpesApro bados/IraDocumentosConpesAprobados/tabid/166/D efault.aspx

27. Galeano ME. Diseño de proyectos en la investigación cualitativa. Medellín: Fondo Editorial Universidad EAFIT; 2004.

28. Municipio de Medellín. Concejo de Medellín. Acuerdo 003 de 2007. Por el cual se adopta la Política pública para la prevención de la morbimortalidad por causas cardio-cerebro-vasculares en el municipio de Medellín. [Internet]. [Consultado 2014 may 1]. Disponible en: http://www.concejodemedellin.gov.co/concejo/m21_g allery/8090.pdf

29. Municipio de Medellín. Concejo de Medellín. Acuerdo 044 de 2010. Por el cual se institucionaliza el programa "Canas al aire" Concejo de Medellín. [Internet]. [Consultado 2014 may 1]. Disponible en: http://www.concejodemedellin.gov.co/concejo/m21_g allery/11019.pdf

30. Municipio de Medellín. Concejo de Medellín. Acuerdo 013 de 2001. Por medio del cual se institucionaliza el plan de atención integral a la infancia y se crea el concejo de política de infancia en la ciudad de Medellín. Crónicas Municipales de Medellín 2000-2003;2004.

31. Municipio de Medellín. Concejo de Medellín. Acuerdo 014 de 2004. Por medio del cual Se crea el Programa "Buen Comienzo" para promover el desarrollo integral, diverso e incluyente de los menores de 6 años. Crónicas Municipales de Medellín 2004-2007;2008.

32. Municipio de Medellín. Concejo de Medellín. Acuerdo 058 de 2011. Por el cual se adopta la Política Pública de Atención Integral a la Primera Infancia Buen Comienzo, se desarrolla un Sistema de Atención Integral y se modifica el Acuerdo 14 de 2004. [Internet]. [Consultado 2014 may 1]. Disponible en: http://www.concejodemedellin.gov.co/concejo/m21_g allery/16320.pdf

33. Municipio de Medellín. Concejo de Medellín. Acuerdo 007 de 2003. Por el cual se crea el programa UNIRES Unidades Integrales de Renovación social en el Municipio de Medellín. Crónicas Municipales de Medellín 2000-2003;2004.

34. Municipio de Medellín. Concejo de Medellín. Acuerdo 008 de 2005. Por el cual se crea el programa Comités de Salud Estudiantil en Medellín. [Internet]. [Consultado 2014 may 1]. Disponible en: http://www.concejodemedellin.gov.co/concejo/m21_g allery/10750.pdf

35. Municipio de Medellín. Concejo de Medellín. Acuerdo 076 de 2006. Por el cual se adopta el Plan Estratégico Municipal de Desarrollo Juvenil de Medellín 2007- 2015. [Internet]. [Consultado 2014 may 1]. Disponible en: http://www.concejodemedellin.gov.co/concejo/m21_g allery/8659.pdf

36. Municipio de Medellín. Concejo de Medellín. Acuerdo 022 de 2003. Por medio del cual se expide la Política Pública para las mujeres Urbanas y Rurales del Municipio de Medellín. Crónicas Municipales de Medellín 2000-2003;2004.

37. Municipio de Medellín. Concejo de Medellín. Acuerdo 033 de 2005. Por el cual Se institucionaliza el Plan de Estímulos para la Mujer como Política Pública. [Internet]. [Consultado 2014 may 1]. Disponible en: http://www.concejodemedellin.gov.co/concejo/m21_g allery/10787.pdf

38. Municipio de Medellín. Concejo de Medellín. Acuerdo 018 de 2001. Por el cual se adopta la política pública para la población de la tercera edad en Medellín. Crónicas Municipales de Medellín 2000-2003;2004.

39. Municipio de Medellín. Concejo de Medellín. Acuerdo 086 de 2006. Por el cual se institucionaliza el Foro Anual del Adulto Mayor en Medellín. [Internet]. [Consultado 2014 may 1]. Disponible en: http://www.concejodemedellin.gov.co/concejo/m21_g allery/8294.pdf

40. Municipio de Medellín. Concejo de Medellín. Acuerdo 002 de 2001. Por el cual acondiciónese las estaciones del Metro para que sean accesibles a personas con discapacidad. Crónicas Municipales de Medellín 20002003;2004.

41. Municipio de Medellín. Concejo de Medellín. Acuerdo 033 de 2004. Por el cual se institucionaliza la "Semana 
de la Discapacidad". Crónicas Municipales de Medellín 2004-2007;2008.

42. Municipio de Medellín. Concejo de Medellín. Acuerdo 053 de 2007. Por el cual se crea el Programa "Apoyo técnico para la Movilidad" en procura de la integración de las personas con discapacidad. [Internet]. [Consultado 2014 may 1]. Disponible en: http://www.concejodemedellin.gov.co/concejo/m21_g allery/7855.pdf

43. Municipio de Medellín. Concejo de Medellín. Acuerdo 086 de 2009. Por el cual se adopta la política pública en discapacidad para el municipio de Medellín. [Internet]. [Consultado 2014 may 1]. Disponible en: http://www.concejodemedellin.gov.co/concejo/m21_g allery/6277.pdf

44. Municipio de Medellín. Concejo de Medellín. Acuerdo 050 de 2006. Por el cual se prohíbe el consumo de cigarrillo, tabaco y sus derivados en los establecimientos oficiales municipales de atención al público, en oficinas estatales del municipio y en edificios de propiedad del Municipio de Medellín. [Internet]. [Consultado 2014 may 1]. Disponible en: http://www.concejodemedellin.gov.co/concejo/m21_g allery/11375.pdf

45. Municipio de Medellín. Concejo de Medellín. Acuerdo 038 de 2005. Por el cual se establece la Política Pública de Seguridad y Soberanía Alimentaria y Nutricional en Medellín. [Internet]. [Consultado 2014 may 1]. Disponible en: https://www.medellin.gov.co/irj/go/km/docs/wpccon tent/Sites/Subportal\%20del\%20Ciudadano/Planeaci \%C3\%B3n\%20Municipal/Secciones/Publicaciones/Do cumentos/PlanAbastecimiento/03\%20Acuerdo $\% 20 \mathrm{M}$ unicipal\%20038\%20-2005\%20Vf.docx.

46. Municipio de Medellín. Concejo de Medellín. Acuerdo 056 de 2008. Por el cual se crea el programa de recuperación nutricional en la ciudad de Medellín. Crónicas Municipales de Medellín 2008-2011;2012.

47. Municipio de Medellín. Concejo de Medellín. Acuerdo 013 de 2003. Por el cual se implementa en el Concejo de Medellín, el programa Participación interdisciplinaria de investigación en temas de ciudad. Crónicas Municipales de Medellín 2000-2003; 2004.

48. Municipio de Medellín. Concejo de Medellín. Acuerdo 018 de 2003. Por el cual Se implementa en forma permanente el Programa Medellín Saludable. Crónicas Municipales de Medellín 2000-2003; 2004.

49. Municipio de Medellín. Concejo de Medellín. Acuerdo 021 de 2004. Por el cual se crea la Red de atención de urgencias para el municipio de Medellín. Crónicas Municipales de Medellín 2004-2007; 2008.

50. Municipio de Medellín. Concejo de Medellín. Acuerdo 059 de 2008. Por el cual se crea en el Municipio de Medellín las "Unidades Móviles de Salud". [Internet]. [Consultado 2014 may 1]. Disponible en: http://www.concejodemedellin.gov.co/concejo/m21_g allery/3893.pdf

51. Municipio de Medellín. Concejo de Medellín. Acuerdo 072 de 2006. Por el cual se institucionaliza la Semana del Deporte La Recreación y La Lúdica. Crónicas Municipales de Medellín 2004-2007;2008.
52. Municipio de Medellín. Concejo de Medellín. Acuerdo 084 de 2009. Por el cual se reglamenta el Sistema de Bicicletas en la ciudad de Medellín que hará parte del Plan de movilidad de la ciudad. [Internet]. [Consultado 2014 may 1]. Disponible en: http://www.concejodemedellin.gov.co/concejo/m21_g allery/6397.pdf

53. Muller P. Elementos para una estrategia de investigación sobre políticas públicas. Innovar. 2000;16:49-54.

54. Comisión Económica para América Latina y el Caribe (CEPAL). América Latina y el Caribe: una mirada al futuro desde los Objetivos de Desarrollo del Milenio. Informe regional de monitoreo de los Objetivos de Desarrollo del Milenio (ODM) en América Latina y el Caribe. Santiago: Naciones Unidas; 2015.

55. Silva FA y cols. Enfermedad cerebro-vascular en Colombia. Revista Colombiana de Cardiología: 2006; 13(2):85-89.

56. Mosquera W, Durán AE, Catillo VR, Castro JM, Karl G, Suárez MM, et al. Guía para la promoción y prevención de la salud cardiovascular desde la infancia. Rev. Colomb. Cardiol. 2009;16(3):162-181.

57. García HI, Giraldo CA y cols. Treinta años de homicidios en Medellín, Colombia, 1979-2008. Cad. Saúde Pública. 2012;28(9):1699-1712.

58. Vargas Velázquez A. El estado y las políticas públicas. Almudena Editores; 1999.

59. Stoker RP. Un marco de análisis para el régimen de implementación: cooperación y reconciliación entre imperativos federalistas. En: Aguilar Villanueva LF. La implementación de las políticas. 3a ed. México D.F: Miguel Ángel Porrúa; 2000;373-412

60. Arroyave Álzate S. Las políticas públicas en Colombia. Insuficiencias y desafíos. Revista del departamento de Ciencia Política. 2011;(1):95-110

61. Roth Deubel AN. Políticas públicas: Formulación, implementación y evaluación. Bogotá: Ediciones Auroras; 2002.

62. González M. Las políticas públicas. Carácter y condiciones vinculantes. En Polis. Investigación y Análisis Sociopolítico y Psicosocial. [Internet]. [Consultado 2016 ene 30]. (Disponible en http://www.juridicas.unam.mx/publica/librev/rev/po lis/cont/20001/pr/pr3. Pdf.

63. Suárez J. Las enfermedades cardiovasculares, gestión de sistemas subnacionales de salud y recomendaciones para plan de acción. Revista Cubana de Salud Pública. 2011;37(2):154-158. 